\title{
Role of Water Layer on Hydrophobic and Hydrophilic Self-Assembled Monolayer Surface
}

\section{Bhattacharyya $\mathrm{T}^{*}$}

Ulsan National Institute of Science and Technology, Eonyang-eup, Ulju-gun, Ulsan, Republic of Korea

${ }^{*}$ Corresponding author: Bhattacharyya T, Ulsan National Institute of Science and Technology, 50 UNISTgil, Eonyang-eup, Ulju-gun, Ulsan, Republic of Korea 689-798, E-mail: tbhattacharyya2010@gmail.com

Citation: Bhattacharyya T (2016) Role of Water Layer on Hydrophobic and Hydrophilic Self-Assembled Monolayer Surface. J Mater Sci Nanotechnol 4(1): 102. doi: 10.15744/2348-9812.4.102

Received Date: August 21, 2015 Accepted Date: January 26, 2016 Published Date: January 28, 2016

\begin{abstract}
The water layer adjacent to the hydrophobic and hydrophilic moieties of self-assembled monolayer surface plays an important role on formation of densely packed self-assembled monolayers. Fourier transforms infrared spectroscopy and atomic force microscopy based study shows that the quality of self-assembled monolayer of thiols is dependent on re-structuring of surrounded water molecules.
\end{abstract}

Keywords: Self-assembled monolayers; Hydrophobicity; Clustering

\section{Introduction}

Self-assembly is a spontaneous but ordered arrangement of smaller building blocks [1]. During this process, the subunits combine in such a way that they finally form a constrained structure like self-assembled monolayers (SAMs) [2]. The arrangement of such fundamental building block in which intermolecular forces play a key role adsorbed on solid surfaces can be spontaneously formed from solution. The building block consist a head group, a specific terminal group and the main hydrophobic chain. The head group which co-valently links the hydrocarbon chain to the semiconductor or metallic surface is responsible for the selfassembly process $[3,4]$ on each type of substrate. The van der Waals and hydrophobic forces interactions among the hydrocarbon chains and also the solvent environment [5-8] ensure a compact packing of the monolayers and stabilize the structure. Among the chemisorbed ordered-monolayers, n-alkanethiols adsorbed on semiconductor or metallic surfaces [9] are a most popular combination. Generally, these monolayers are deposited by spontaneous adsorption from ethanol solutions. In this study, the role of water in formation of densely packed SAMs of 16-Mercaptohexadecanoic acid (MHDA) thiol has been described.

\section{Materials and Methods}

\section{Monolayer Preparation}

Self-assembled monolayers of 16-Mercaptohexadecanoic acid (MHDA) thiol [10] purchased from sigma aldrichhas been prepared for this study. SAMs prepared on planar substrates like thin films of gold supported on silicon wafers in this case. These substrates are easy to prepare by physical vapor deposition (PVD) method. The resulting substrate is then immersed into a $2 \mathrm{mM}$ solution of MHDA. The self-assembly time used to prepare SAMs on this substrate was 2 minutes.

\section{Fourier transform infrared (FT-IR) spectroscopy (TENSOR II FT-IR Spectrometer with HYPERION 3000 FT-IR Microscope) measurement}

To investigate the effect of water on SAMs, samples were prepared in both ethanol and ethanol-water mixture in a ratio of 7:3, 3:2 and 1:1 respectively and was used in FTIR measurements under nitrogen $\left(\mathrm{N}_{2}\right)$ with low signal attenuation.

\section{Fourier transform infrared (FT-IR) mapping measurement (TENSOR II FT-IR Spectrometer with HYPERION 3000 FT-IR Microscope)}

The FTIR mapping, samples were kept in microscope chamber under nitrogen $\left(\mathrm{N}_{2}\right)$ with low signal attenuation. The estimated surface area probed with the IR beam was $30 \mu \mathrm{M}$ during the experiment.

\section{Atomic force microscopy (AFM) based study}

The measurements were carried out with Nano Scope III AFM in the taping mode. AFM scans were performed in air and ethanol. Silicon nitride cantilevers with nominal spring constants of 0.38 and $0.12 \mathrm{~N} / \mathrm{m}$ were used. The system was allowed to equilibrate prior to taking measurements. All images shown in this work correspond to unprocessed data obtained in the height mode. 


\section{Results and Discussion}

The Fourier transform infrared (FTIR) spectroscopy data (see Figure 1) suggests that the band responsible for asymmetric CH2 stretching vibration of SAMs appears in between $2917-2922 \mathrm{~cm}^{-1}$ and in all cases but the position varies from one sample to the other. The adsorption band at $2850 \mathrm{~cm}^{-1}$ is due to symmetric $\mathrm{CH}_{2}$ stretching vibration of SAMs and it indicates the ordering by lateralinteraction of SAMs. The band position varies with the ordering of alkyl chains. The peak is observed at $2921.4 \mathrm{~cm}^{-1}$ when the monolayer has been prepared in degassed ethanol but the position changes to $2919.5 \mathrm{~cm}^{-1}, 2917.5 \mathrm{~cm}-1$ and $2917.5 \mathrm{~cm}^{-1}$ when the SAM has been prepared in diluted degassed ethanol upon incorporation of degassed deionized (DI) water in a ratio of 7:3 $(\mathrm{v} / \mathrm{v}), 3: 2(\mathrm{v} / \mathrm{v})$ and 1:1 (v/v) respectively as shown in Figure 1. An increase in absorbance intensity is observed when SAM has been prepared in degassed ethanol-water mixture. The absorbance is least when the solvent was ethanol only and highest when ethanol was diluted with water in 1:1 (v/v) ratio. The low frequency shift of the asymmetric $\mathrm{CH}_{2}$ band indicates the enhancement of monolayer order [11]. The high frequency shift tells the formation of multilayer structure due to stacking of multiple monolayers. Abroadening in asymmetric band is observed [11] but it is narrower for monolayers.

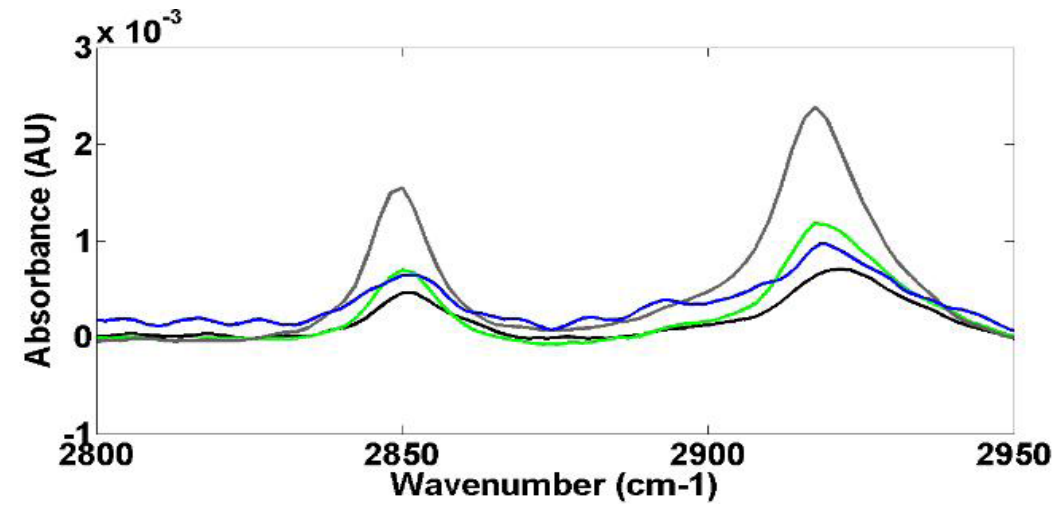

Figure 1:Transmission FTIR spectra of SAMs grown in ethanol (black) and ethanol diluted with degassed DI water in a ratio of 7:3 (blue), 3:2 (green) and 1:1 (violet)

The uniform blue pixel intensity which is reflected from FT-IR grid measurement gives an important clue on uniform formation of SAMs. In some region it is monolayer (see Figure 2(b)) and in some regions it contains two layers (see Figure 2(b)) or even multilayers (see Figure 2(a)). It depends on how the clustering of SAMs due to multiple van der Waals force of attractionis controlled by surrounded water structure (see explanations later). The increase in absorbance intensity can be due to the precipitation of thiols. To nullify the possibility of thiol precipitation, atomic force microscopy (AFM) study has been carried out. From Figure 3 it is clear that the roughness of the wafer increases upon thiolation and it increases up to ethanol to water ratio 7:3 but the roughness decreases again with further incorporation of water up to 1:1 ratio. A very high increase in roughness with the ratio 3:7 $(\mathrm{v} / \mathrm{v})$ ratio confirm that thiols get precipitated with this very high water content. Interestingly the roughness with this particular ratio (1:1 ratio) is comparable with ethanol only and it is also clear from Figure 3 and 4 as well. As the dimensions of thin layer SAMs are typically in nanometer level therefore to understand the organization and quality of SAMs roughness of surface is more important factor. Because thickness can tell only about the precipitation issue but roughness serves both the precipitation and quality control of SAMs. The response on SAM formation with 1:1 (v/v) ratio is very interesting. Thiols however, can arrange themselves around water without breaking hydrogen bonds or losing much energy and the hydrophobic molecule can interact with these water molecules with multiple van der Waals interactions, due to the small size of water molecules and flexibility in their spatial arrangement.

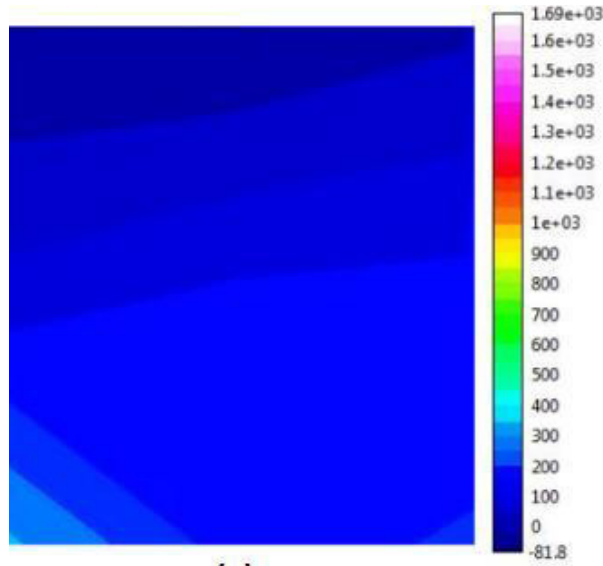

(a)

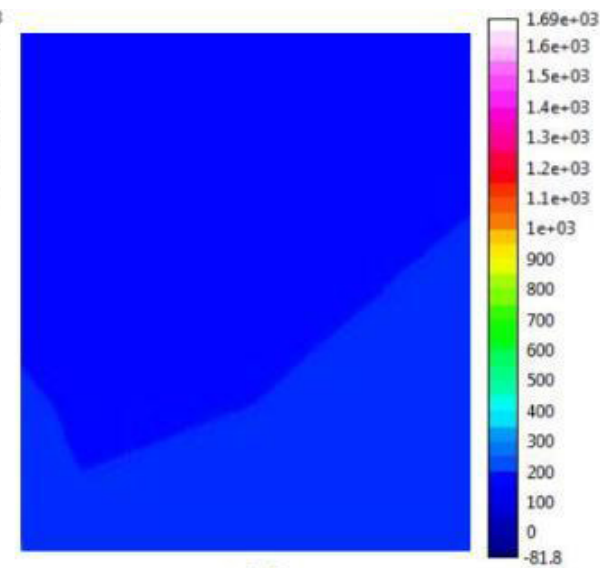

(b)

Figure 2: FTIR mapping of SAMs (a) blue color represents the SAMs and the cyan color is the region without SAMson Si wafer (b) FTIR mapping of SAMs fully covered on Si wafer 

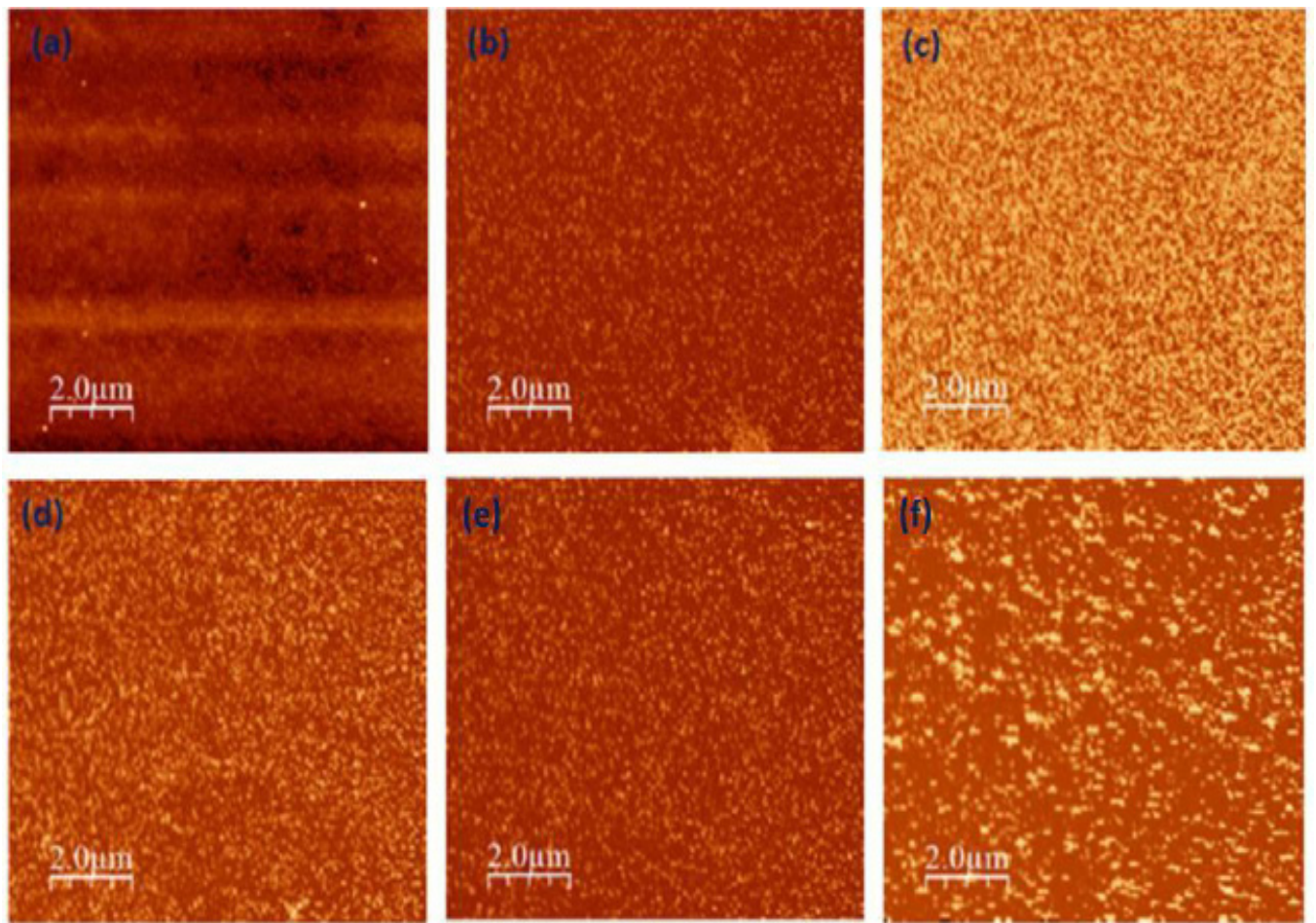

Figure 3: AFM images of (a) Si wafer without thiolation and after thiolation with (b) ethanol only, diluted ethanol with degassed water taken in ratio (c) 7:3, (d) 3:2, (e) 1:1 and (f) 3:7 (v/v) respectively

The "hydrophobic effect" of ethanol dissolved thiols in water is primarily a consequence of changes in the clustering in the surrounding water rather than water-solute interactions. This $1: 1(\mathrm{v} / \mathrm{v})$ ratio provides the best close packed cluster structure due to 1:1 interaction. In all other ratio either hydrophobic or hydrophilic forces dominates but in this ration the clustering is stabilized by 1:1 water-solute interaction. The long alkyl chains associate via van der Waals and hydrophobic interactions in aqueous solutions that promote organization of the stable SAMs.

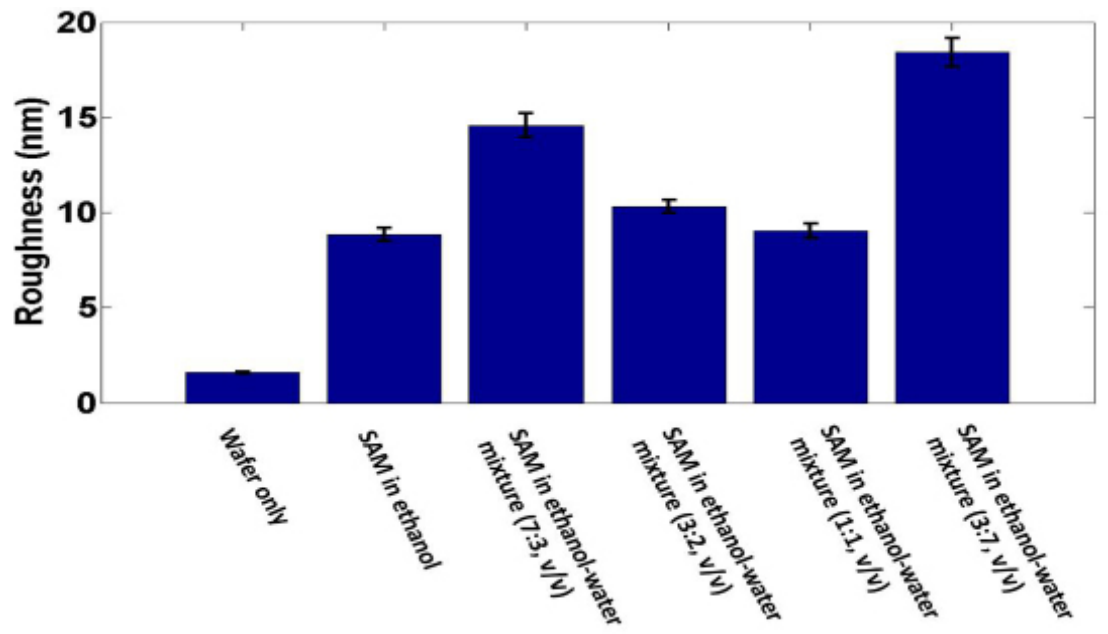

Figure 4: Roughness calculated from AFM images of wafer only without thiolation and after thiolation in degassed ethanol only and degassed ethanol-DI water taken in ratio (c) 7:3 (v/v), (d) 3:2 (v/v), (e) 1:1 (v/v) and (f) 3:7 (v/v) respectively

Initially when ethanol dissolved thiols are surrounded with water molecules the assembly of $n$-alkane thiols experience strong intermolecular forces between the alkane chains due to hydrophobic interaction as well as change the clustering in the surrounding water result ordering of SAMs. The increased absorbance in Figure 1 indicates the formation of densely packed monolayers. When the water content is less, the change in clustering of thiols in the surrounding water is less and moreover the randomness of the alkane chains are higher. It creates some local disorder result increase in roughness but when the water content is increases a bit the alkane chains are densely packed due to multiple van der Waals force of interaction. As a result the hydrophobic effect spatially rearrange the thiols to a densely packed cluster. So, the quality of self-assembled monolayer is strongly dependent on surrounded water structure. The roughness of the surface also decreases as it is reflected in Figure 3 and 4 . But when water content is very high (i.e. ethanol to water ratio 3:7, v/v) the clustering effect collapses due to less solubility of thiols in the mixtures. A disorder is created due to precipitation of thiols. Therefore an abrupt increase in roughness (also the surface topology changes significantly) is observed and most interestingly, ethanol to water 1:1 (v/v) ratio performs the best for SAM formation. 


\section{Conclusion}

Therefore from this observation it can be said that water plays an important role on clustering of SAMs due to multiple van der Waals force of attraction. This clustering effect is found to be dependent on nature of the solvent. Ethanol to water ratio 1:1 (v/v) is found to be the best for rearrangement of thiols to a densely packed cluster.

\section{References}

1. Wilbur JL, Whitesides GM (1999) Nanotechnology, New York.

2. Gates BD, Xu Q, Stewart M, Ryan D, Willson CG, et al. (2005) New approaches to nanofabrication: molding, printing, and other techniques. Chem Rev 105: 1171-96.

3. Vericat C, Vela ME, Benitez G, Carrob P, Salvarezza RC (2010) Self-assembled monolayers of thiols and dithiols on gold: new challenges for a well-known system. Chem Soc Rev 39: 1805-34.

4. Dubois LH, Nuzzo RG (1992) Synthesis, Structure, and Properties of Model Organic Surfaces. Annu Rev Phys Chem 43: 437-63.

5. Bain CD, Troughton EB, Tao YT, Evall J, Whitesides GM, et al. (1989) Formation of monolayer films by the spontaneous assembly of organic thiols from solution onto gold. J Am Chem Soc 111: 321-35.

6. Sur UK, Lakshminarayanan V (2004) A study of the hydrophobic properties of alkanethiol self-assembled monolayers prepared in different solvents. J Electroanal Chem 565: 343-50.

7. Canaria CA, Maloney JR, Yu CJ, Smith JO, Fraser SE, et al. (2006) Formation of Biotinylated Alkyl thiolate Self-Assembled Monolayers on Gold. Lab Chip 6: 289-95.

8. Dai J, Li Z, Jin J, Cheng J, Kong J, et al. (2008) Study of the solvent effect on the quality of dodecanethiol self-assembled monolayers on polycrystalline gold. J Electroanal Chem 624: 315-22.

9. Yan D, Jordan JL, Burapatana V, Jennings GK (2003) Formation of n-Alkanethiolate Self-Assembled Monolayers onto Gold in Aqueous Micellar Solutions of n-Alkyltrimethylammonium Bromides. Langmuir 19: 3357-64.

10. Ulman A (1996) Formation and Structure of Self-Assembled Monolayers. Chem Rev 96:1533-54.

11. Tao YT, Hietpas GD, Allara DL (1996) HCl vapor-induced structural rearrangements of $n$-alkanoate self-assembled monolayers on ambient silver, copper, and aluminum surfaces. J Am Chem Soc 118: 6724-35.

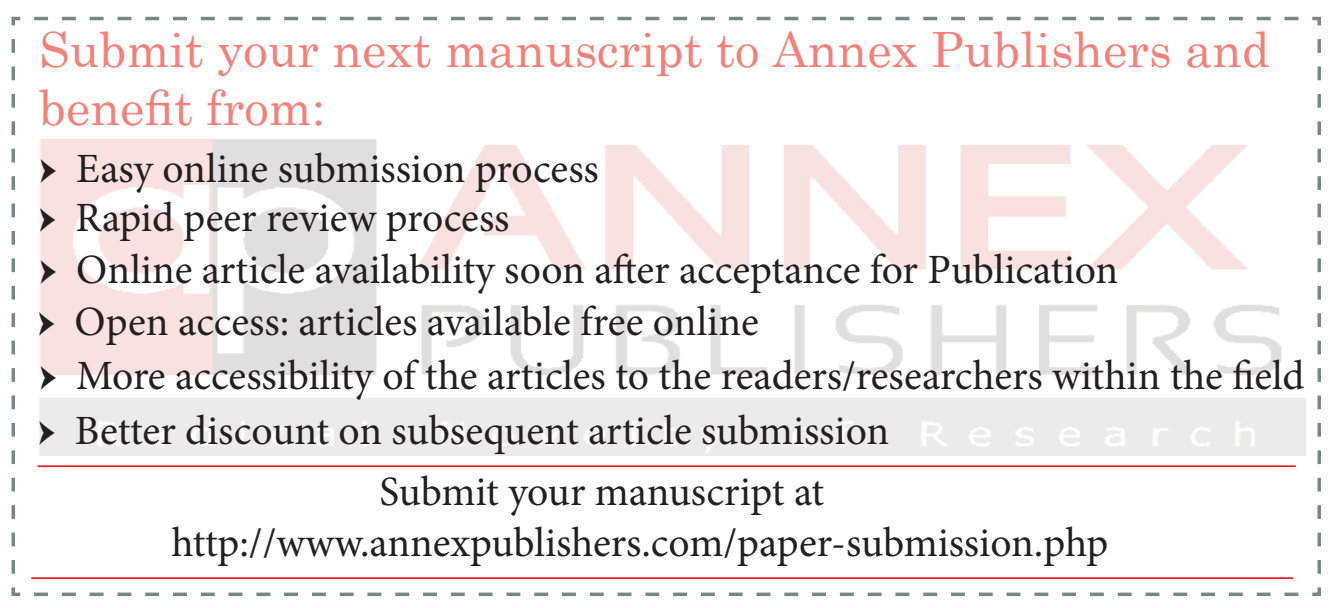

